\title{
Neuro-retinitis asociada a enfermedad por arañazo de gato: Presentación de dos casos y revisión de la literatura
}

\author{
Jorge Pérez G., José M. Munita S., Rafael Araos B., Juan P. López G., Ricardo Stevenson A., \\ Patricia González A., Daniel Pérez C. y Luis M. Noriega R.
}

\section{Cat scratch disease associated neuroretinitis: Clinical report and review of the literature}

Cat scratch disease is the main clinical presentation of Bartonella henselae infection. However, ocular manifestations of bartonellosis occur in about 5 to $10 \%$ of the patients, mainly presenting as neuroretinitis, choroiditis or oculoglandular syndrome of Parinaud. We describe two patients with documented $B$. henselae infection and typical ocular compromise. Both patients were treated and had a favorable visual outcome.

Key words: Ocular bartonellosis, neuroretinitis, Bartonella henselae.

Palabras clave: Bartonella henselae, neuro-retinitis, bartonelosis ocular.

\section{Introducción}

Las manifestaciones clínicas que produce la infección por Bartonella henselae son diversas, siendo más común la denominada "enfermedad por arañazo de gato" (EAG). Ésta se caracteriza por la presencia de una pápula en el sitio de inoculación, seguida de adenopatías regionales y fiebre de intensidad variable $e^{1,2}$.

Entre 5 y $10 \%$ de los pacientes con EAG tienen compromiso ocular, manifestado como síndrome óculoglandular de Parinaud, neuro-retinitis y retino-coroiditis focal $^{3-5}$. El compromiso retiniano es poco frecuente y el diagnóstico debe plantearse ante un cuadro clínico sugerente y serología positiva para $B$. henselae . $^{\text {. }}$

A continuación presentamos dos pacientes con infección aguda por $B$. henselae documentada serológicamente, ambos con compromiso ocular, los que recibieron tratamiento y lograron recuperación completa de la visión.

\section{Caso 1}

Paciente de sexo masculino, 30 años de edad. Portador de enfermedad celíaca, en tratamiento con régimen sin gluten. Consultó por fiebre de tres días de evolución, que cedió con antipiréticos en $24 \mathrm{~h}$. Luego de estar cuatro días asintomático, comenzó con fiebre intermitente y leve compromiso del estado general. Fue evaluado 14 días después del inicio de los síntomas, constatándose en buen estado general y un examen físico normal. Se solicitó hemograma, proteína $\mathrm{C}$ reactiva $(\mathrm{PCR})$ y radiografía de tórax, los cuales resultaron normales.
A la tercera semana de evolución presentó bruscamente visión borrosa del ojo izquierdo, que progresó en 12 horas a la ceguera completa, hospitalizándose para estudio y manejo.

Entre sus antecedentes personales destacó que no fumaba ni usaba drogas ilícitas, consumía alcohol en forma ocasional, no había viajado al exterior en el último año y no refería factores de riesgo para enfermedades de transmisión sexual. Sin antecedentes familiares relevantes. En su casa tenía un gato como mascota, aunque no refería el antecedente de haber sido arañado por éste.

En el examen físico de ingreso se encontraba en buen estado general, febril $38^{\circ} \mathrm{C}$, sin adenopatías. La piel no presentaba lesiones compatibles con un sitio de inoculación. El examen de cabeza, cuello, tórax, abdomen y extremidades era normal.

Examen oftalmológico: agudeza visual $(\mathrm{AV})$ de ojo derecho $(O D)=20 / 20$; ojo izquierdo $(O I)=20 / 400$, con fijación excéntrica por escotoma central que no corrigió con refracción. La presión intraocular fue $14 \mathrm{~mm} / \mathrm{Hg}$ en ambos ojos, los reflejos pupilares impresionaron normales, sin defecto pupilar aferente relativo (DPAR), la motilidad ocular estaba conservada y el examen biomicroscópico fue normal. En el fondo de ojos se observó edema de papila bilateral, mayor en OI, acompañado de edema macular y lesiones blanquecinas profundas retinocoroídeas, en algunas zonas de aspecto intravascular (Figura 1).

Laboratorio inicial: Hemograma: hematocrito 43\%, leucocitos: $11.900 / \mathrm{mm}^{3}$ (neutrófilos 63\%, linfocitos 19\%, monocitos 7\%, baciliformes $9 \%$ ) y VHS $36 \mathrm{~mm} / \mathrm{h}$; PCR: $6,4 \mathrm{mg} / \mathrm{dL}$; perfil bioquímico normal; hemocultivos (-),
Clínica Alemana, Santiago, Chile.

Departamento de Medicina Interna (JPG, JMMS, RAB, LMNR)

Servicio de Oftalmología (JPLG, RSA) Laboratorio de Microbiología (PGA) Hospital Padre Hurtado, Santiago, Chile

Servicio Medicina Interna (JPG, JMMS, RAB),

Facultad de Medicina Clínica

Alemana-Universidad del Desarrollo (JPG, JMMS, RAB, JPLG, RSA, PGA, DPC, LMNR)

Recibido: 9 de abril de 2010 Aceptado: 12 de agosto de 2010

Los autores no tienen conflictos de interés que reportar.

\section{Correspondencia a:} Luis Miguel Noriega Ricalde Inoriega@alemana.cl 
VDRL (-). Tomografía computada cerebral y ecocardiograma transesofágico sin hallazgos patológicos.

El estudio serológico inicial resultó negativo para: B. henselae (IgG por ELISA), citomegalovirus, VIH, Brucella spp., virus de Epstein-Barr, Borrelia burgdorferi,

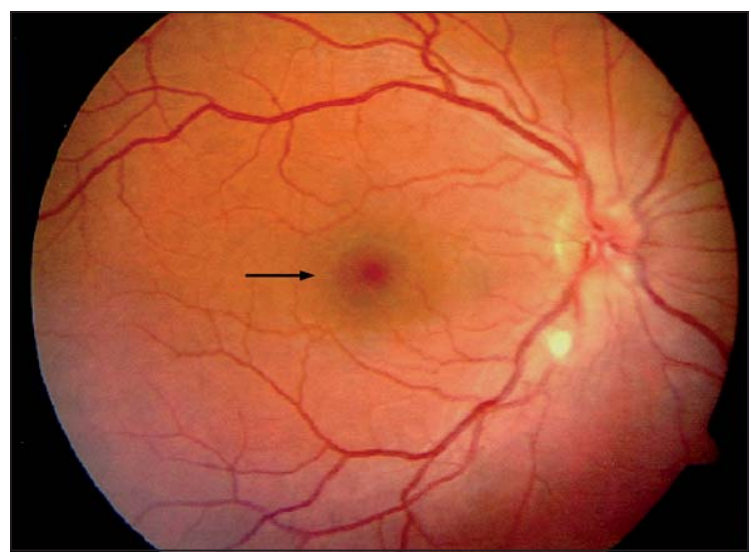

Toxoplasma gondii, virus herpes simplex 1 y 2 , virus de hepatitis A, B y C. Además se solicitó PPD, estudio de autoinmunidad (ANA, anti-DNAdh, C3 y C4) y un estudio citoquímico de LCR, todos normales.

Ante la exploración inicial no concluyente y debido a que se mantenía una alta sospecha clínica, se repitió el estudio de $B$. henselae una semana después, esta vez con técnica de inmunofluorescencia indirecta (IFI), resultando positivo (título $\geq 1 / 256$ ). Con este hallazgo se realizó tratamiento con doxiciclina $100 \mathrm{mg} \mathrm{c} / 12 \mathrm{~h}$ y rifampicina $300 \mathrm{mg} \mathrm{c} / 12 \mathrm{~h}$, vía oral durante 6 semanas. A las 24 h de antibioterapia desapareció la fiebre y se demostró una disminución progresiva del edema papilar, con lenta recuperación de la visión del ojo izquierdo. El fondo de ojo control veinte días después evidenció la presencia de una imagen de estrella macular, conformando el cuadro clínico clásico de neuroretinitis por B. henselae (Figura 2).

En controles sucesivos se comprobó la regresión casi completa del edema de papila y macular, con mejoría progresiva de la visión del OI (hasta 20/30) y desaparición del escotoma central (Figura 3).
Figura 1. Caso 1. Fotografía de fondo de ojo al ingreso, mostrando Ol con edema de papila, edema macular y lesiones retinocoroídeas blanquecinas (ver flecha).
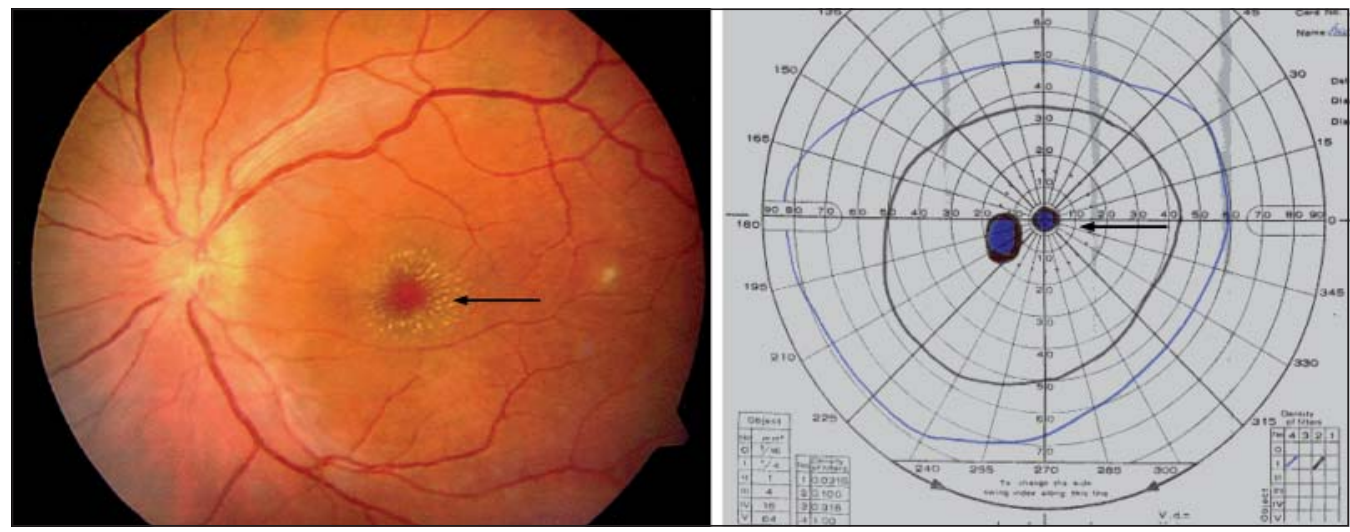

Figura 2. Caso 1. Fotografía de fondo de ojo (a) y campo visual Goldman (b) al mes de evolución. Ol: Edema de papila en regresión; estrella macular y escotoma central en campo visual (ver flechas).

Figura 3. Caso 1. Fotografía de fondo de ojo (a) y campo visual Goldman (b), control. Ol: Regresión de edema de papila y estrella macular (ver flecha); campo visual con resolución del escotoma.
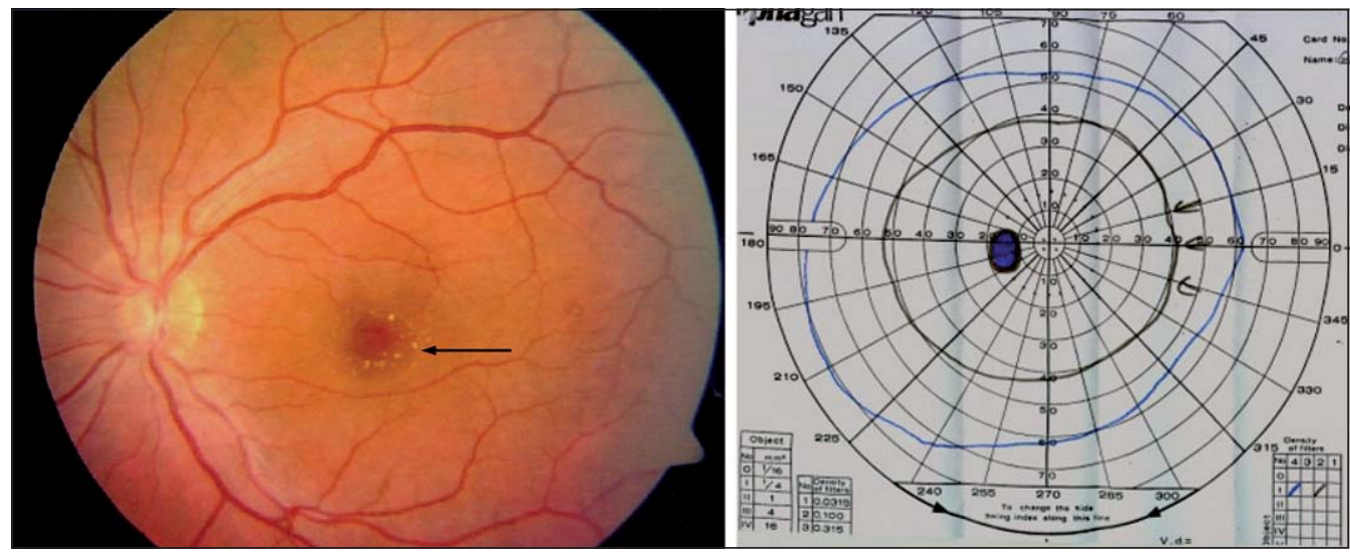


\section{Caso 2}

Paciente de sexo masculino, edad 33 años, con antecedentes de meningitis meningocóccica en la infancia, sin secuelas, y una neumonía grave cinco años antes. Se hospitalizó por un cuadro de dos días de evolución, caracterizado inicialmente por cefalea de inicio insidioso, de predominio frontal e intensa, y posteriormente fiebre hasta $38^{\circ} \mathrm{C}$, con calofríos. En la revisión por sistemas refería fotofobia. Los signos vitales eran estables, no se encontraron hallazgos relevantes en el examen físico segmentario y el examen neurológico fue normal. En el hemograma un recuento de leucocitos era normal con $10 \%$ de baciliformes, VHS y PCR normal. En el perfil bioquímico había solamente una leve elevación de las transaminasas (GOT 39 U/L, GPT 62 U/L). El estudio de imágenes (radiografía de tórax, ecografía de abdomen y resonancia magnética de cerebro) no encontró alteraciones significativas. Se controló la cefalea con anti-inflamatorios y luego de $48 \mathrm{~h}$ fue dado de alta en buenas condiciones, con diagnóstico de infección viral inespecífica.

Evolucionó en buenas condiciones durante una semana, hasta que volvió a consultar por la aparición de dolor y aumento de volumen en la región pre-auricular izquierda, asociado a fiebre hasta $38^{\circ} \mathrm{C}$, compromiso del estado general, fotofobia y disminución de la agudeza visual. Se hospitalizó nuevamente para estudio. En el interrogatorio dirigido, esta vez aportó el antecedente de tener gatos como mascotas.

$\mathrm{Al}$ examen físico de ingreso el paciente estaba febril $38^{\circ} \mathrm{C}$, con aumento de volumen parotídeo izquierdo, donde además se palpaban adenopatías de aspecto inflamatorio. En la piel de las extremidades superiores se veían múltiples lesiones que el paciente atribuyó a arañazos de gato. El resto del examen segmentario no tenía alteraciones.

En el examen oftalmológico destacaba un fondo de ojo con edema de papila bilateral, hemorragias en astilla, mayor a derecha, con mácula plana en ambos ojos. Se realizó una ecografía cervical que demostró la presencia de numerosas adenopatías intra-parotídeas derechas. Los exámenes generales fueron normales y el estudio serológico descartó infección por VIH, T. gondii, sífilis, toxocariasis, primoinfección citomegálica y por virus de Epstein-Barr. Se realizó una IFI para $B$. henselae que resultó positiva (título $\geq 1 / 256$ ) y una ecografía parotídea con adenopatías compatibles con EAG (Figura 4). En el examen oftalmológico de control realizado durante la hospitalización, persistía el edema de papila bilateral y se hizo evidente una mínima estrella macular en el ojo derecho. Se inició tratamiento con doxiciclina y rifampicina que recibió sin problemas durante ocho semanas. Junto a éste, se observó control del cuadro inflamatorio sistémico y desaparición de las adenopatías. La evolución

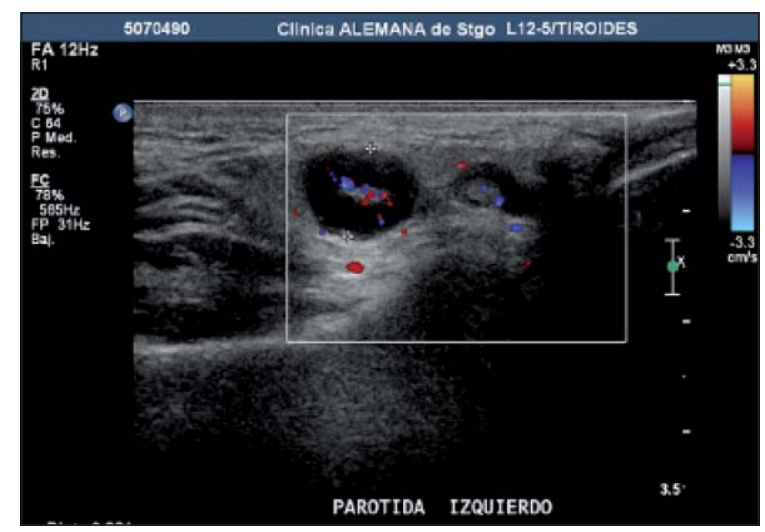

oftalmológica posterior ha sido lenta, con un último seguimiento a los dos meses. Se logró demostrar regresión casi completa del edema papilar y desaparición de la estrella macular derecha. Sin embargo, la agudeza visual no se ha recuperado ad integrum.

\section{Discusión}

La EAG fue descrita como entidad clínica hace medio siglo, pero a pesar de sospecharse una causa infecciosa desde el comienzo, la relación con $B$. henselae fue establecida recién en 1983, cuando mediante tinción de Warthin-Starry se demostró la presencia de formas bacilares en ganglios linfáticos de 29 de 34 pacientes con $\mathrm{EAG}^{7}$. Este agente es un bacilo gramnegativo pleomórfico que requiere medios enriquecidos para su crecimiento, pudiendo tardar hasta cuatro semanas en desarrollarse ${ }^{8}$. $\mathrm{Su}$ hospedero definitivo es el gato, siendo el hombre un hospedero accidental. La transmisión se produce por inoculación del microorganismo a través de un arañazo o mordida de gato, y existen reportes que sugieren transmisión por picaduras de pulgas de gatos ${ }^{9}$. La presentación más típica es un cuadro sistémico, autolimitado, que se caracteriza por fiebre y adenopatías (cervical y axilar) conocido como $\mathrm{EAG}^{10}$. En E.U.A. afecta aproximadamente a 22.000 personas al año ${ }^{11}$, no existiendo en nuestro medio cifras de incidencia. La prevalencia estimada en base a estudios poblacionales pequeños realizados en Chile es de 10 a $13 \%{ }^{12}$.

El compromiso ocular puede producirse por vía sistémica o por inoculación del microorganismo a las conjuntivas y las presentaciones clínicas son variadas y de diferente gravedad ${ }^{13-15}$. La manifestación ocular generalmente sucede a las sistémicas, pudiendo presentarse en ausencia de síntomas generales ${ }^{16}$. Las formas oculares más frecuentes son el síndrome óculo-glandular de Parinaud, los puntos blancos retino-coroideos, el edema de papila y la estrella macular ${ }^{14,17}$.
Figura 4. Caso 2. Ecografía de ganglio parotídeo. 
El compromiso neuro-retiniano es la segunda forma más frecuente de presentación de la infección ocular por $B$. henselae, siendo la primera el síndrome de Parinaud. La neuro-retinitis clásica se caracteriza por disminución o pérdida aguda uni o bilateral de la visión, asociada a defecto pupilar aferente, alteración del campo visual y edema del disco óptico, con extensión al área peripapilar y macular ${ }^{15}$. Al reabsorberse el componente seroso del edema se produce precipitación de lípidos en la capa plexiforme externa de la retina, disponiéndose en una forma estrellada característica ${ }^{18}$ (Figura 2). Estos fenómenos fueron descritos ya en 1916 por Leber ${ }^{19,20}$. En formas atípicas es posible observar edema de papila, inicialmente sin la correspondiente estrella macular, con buena visión y sin defecto pupilar aferente ${ }^{14}$, como ocurrió con nuestro segundo caso.

El diagnóstico etiológico no es fácil del punto de vista clínico, ya que el aspecto en el examen de fondo de ojos es común a la mayoría de las neuro-retinitis ${ }^{21,22}$, cuyas posibilidades etiológicas se señalan en la Tabla 1.

Para tener una aproximación a este cuadro clínico inespecífico, debe efectuarse una detallada anamnesis precisando antecedentes de viajes, contactos sexuales, alimentos y la presencia de mascotas. En los dos casos reportados, la presencia de contacto frecuente con gatos, ambos con crías menores, orientó al diagnóstico; al igual que la fiebre, que orienta hacia una patología infecciosa.

En la búsqueda de laboratorio se debe incluir además de los exámenes generales, estudio para los diversos agentes que se deben contemplar en el diagnóstico diferencial, como micobacterias, virus de Epstein-Barr, citomegalovirus, T. gondii, Treponema pallidum y B. burgdorferi. También se deben contemplar patologías no infecciosas que pueden simular la enfermedad, como enfermedad de Kawasaki, enfermedad de Kikuchi y sarcoidosis, entre otras (Tabla 1).

La ecografía puede mostrar algunas características sugerentes de la enfermedad, como la presencia de compromiso hepato-esplénico ${ }^{23} \mathrm{y}$, como ocurrió en el caso 2 de este reporte, la presencia de adenopatías de ubicación intra parotídea, consideradas altamente sugerentes de $\mathrm{EAG}^{24}$ (Figura 4).

El estudio microbiológico es complejo y de bajo rendimiento, por tratarse de bacterias nutricionalmente exigentes y de crecimiento lento. En tejidos o líquidos se puede efectuar tinción de Warthin-Starry que permite visualizar el microorganismo o RPC especifica, que tiene alta especificidad y permite identificar las diferentes especies de Bartonella, pero con sensibilidad limitada, descrita entre 43 y $76 \%{ }^{25}$.

La utilidad de los hemocultivos varía de acuerdo con la técnica utilizada y el cuadro de presentación ${ }^{26}$. La sensibilidad para endocarditis por Bartonella sp varía entre 5 y $70 \%$, siendo el subcultivo en líneas celulares (shell-vial) y la incubación con técnica de lisis-centrifugación las de mejor rendimiento ${ }^{8}$. Por otra parte, la sensibilidad de los hemocultivos en el diagnóstico de angiomatosis bacilar se encuentra en torno a $50 \%{ }^{27}$. El rendimiento de los hemocultivos en EAG sin complicaciones es bajo, con $\sim 6 \%$ de sensibilidad ${ }^{8}$.

El diagnóstico serológico se puede hace por técnicas de inmunofluorescencia indirecta (IFI) o por ELISA. La sensibilidad y especificidad de ambas técnicas depende del valor del punto de corte utilizado para considerar el examen como positivo o negativo. En el caso de IgG por IFI, con un punto de corte $\geq 1 / 64$, la sensibilidad y especificidad es de 88 y $99 \%$ respectivamente, levemente superior al rendimiento reportado para ELISA ( 85 y 95\% respectivamente $)^{28}$. En nuestro país, considerando una seroprevalencia de al menos $13 \%$ en la población general, se utiliza un punto de corte más alto $(1 / 256)$ para la IgG por IFI, lo que probablemente permite mantener un especificidad adecuada del examen ${ }^{12}$. En el primer caso de nuestro reporte la serología inicial realizada por ELISA fue negativa, pero las características del cuadro y el resto de los tests negativos motivaron repetir el examen con IFI, la que resultó positiva. Esta discordancia pudiera ser explicada por la diferencia en sensibilidad ya comentada

\begin{tabular}{|c|c|c|c|c|}
\hline Virales & Bacterianas & Parásitos & Hongos & No infecciosas \\
\hline Epstein-Barr & Bartonella henselae & Toxoplasma gondii & Histoplasma sp & Sarcoidosis \\
\hline Varicela-Zoster & Mycobacterium sp & Toxocara sp & & Poliarteritis nodosa \\
\hline Herpes simplex & Salmonella sp & & & Enfermedad inflamatoria intestinal \\
\hline Influenza A & Treponema pallidum & & & Oclusión de vena central de la retina \\
\hline Hepatitis B & Leptospira sp & & & Retinopatía hipertensiva \\
\hline Parotiditis & Borrelia sp & & & Retinopatía diabética \\
\hline \multirow[t]{2}{*}{ Coxsackie B } & & & & Enfermedad de Kikuchi \\
\hline & & & & Enfermedad de Kawasaki \\
\hline
\end{tabular}


y por el tiempo transcurrido entre la toma de ELISA e IFI (una semana) ${ }^{29}$. La determinación de IgM ha mostrado tener menor sensibilidad que la IgG, ya sea con técnica de ELISA o IFI. Por lo tanto, frente a una manifestación clínica sugerente y epidemiología concordante con EAG, y exámenes serológicos negativos, se sugiere siempre repetir la determinación de IgG por IFI, transcurrida al menos una semana.

La sensibilidad de la RPC para enfermedad por arañazo de gato varía entre 66 y 78\% en tejido ganglionar, según sean los criterios utilizados para el diagnóstico. En casos de angiomatosis bacilar y endocarditis, la sensibilidad varía entre 81 y $100 \%$, mientras que la especificidad se acerca a $100 \%{ }^{24,30-32}$.

Al hablar de tratamiento en EAG, se debe considerar que la mayoría de los casos tienen regresión espontánea, en tiempo variable, sin terapia y que no hay un correlato entre la sensibilidad in vitro para los antimicrobianos y su respuesta clínica ${ }^{33}$. En el tratamiento de EAG de presentación ganglionar, los estudios son en general retrospectivos, habiéndose demostrado alguna eficacia con el uso de azitromicina, ciprofloxacina, rifampicina y gentamicina $^{34}$. El único estudio prospectivo randomizado, que compara azitromicina con placebo, favorece la primera en forma clara ${ }^{35}$. La duración recomendada del tratamiento antimicrobiano para EAG es de 5 días $^{33}$. Con respecto a neuro-retinitis, a pesar de no haber estudios controlados disponibles, el tratamiento recomendado es doxiciclina $200 \mathrm{mg}$ al día asociado a rifampicina $600 \mathrm{mg}$ al día durante 4 a 6 semanas. Este esquema parece acortar la evolución de la enfermedad y acelerar la recuperación visual $^{15,36}$. Su ventaja radicaría en la buena penetración de rifampicina al SNC y al aumento de los radicales libres de oxígeno en los polimorfonucleares inducido por las tetraciclinas ${ }^{35}$.

El curso clínico con tratamiento es habitualmente favorable, pero impredecible ${ }^{35}$, con recuperación de la visión en la mayoría de los pacientes, aunque no siempre a los niveles previos a la enfermedad, siendo las complicaciones como obstrucción de ramas de la arteria y vena de la retina o desprendimiento de retina poco frecuentes. No se ha demostrado que el uso de corticosteroides tenga algún impacto en la evolución ${ }^{22}$.

En suma, estos dos casos nos refuerzan la importancia de plantear la infección por $B$. henselae ante la presencia de fiebre y compromiso visual, así como la importancia de preguntar por contacto con mascotas. Además nos enseñan, especialmente en el primer caso, a interpretar y validar los estudios de laboratorio en el contexto clínico.

\section{Resumen}

La infección por Bartonella henselae se presenta característicamente con adenopatías regionales y fiebre de intensidad variable, entidad conocida como "enfermedad por arañazo de gato". Alrededor de 5 a 10\% de los casos desarrolla compromiso ocular, entre los que destacan el síndrome óculo-glandular de Parinaud, la neuro-retinitis y la retino-coroiditis focal. A continuación se presentan dos pacientes con infección aguda por $B$. henselae y compromiso ocular. Ambos recibieron tratamiento y evolucionaron con recuperación completa de la visión.

\section{Referencias}

1.- Smith D L. Cat-scratch disease and related clinical syndromes. Am Fam Physician 1997; 55: 1783-9.

2.- Abarca K, Vial P, Rivera M. Infección por Bartonella henselae en pacientes inmunocompetentes: enfermedad por arañazo de gato. Rev Méd Chile 1996; 124: 1341-9.

3.- Cunningham E T, Koehler J E. Ocular bartonellosis. Am J Ophthalmol 2000; 130: 340-9.

4.- Rothova A, Kerkhoff F, Hooft H J, Ossewaarde J M. Bartonella serology for patients with intraocular inflammatory disease. Retina 1998; 18: 348-55.

5.- Massei F, Gori L, Macchia P, Maggiore G. The expanded spectrum of bartonellosis in children. Infect Dis Clin North Am 2005; 19: 691-711.

6.- Brazis P W, Lee A G. Optic disk edema with a macular star. Mayo Clin Proc 1996; 71: 1162-6.

7.- Wear D J, Margileth A M, Hadfield T L, Fischer G W, Schlagel C J, King F M. Cat scratch disease: a bacterial infection. Science 1983; 221 (4618): 1403-5.

8.- La Scola B, Raoult D. Culture of Bartonella quintana and Bartonella henselae from human samples: a 5-year experience (1993 to 1998). J Clin Microbiol 1999; 37: 1899-905.

9.- Ferrés M, Abarca K, Godoy P, García P, Palavecino E, Méndez G, et al. Presence of Bartonella henselae in cats: natural reservoir quantification and human exposition risk of this zoonoses in Chile. Rev Med Chil 2005; 133 : 1465-71.

10.- Windsor J J. Cat-scratch disease: epidemiology, aetiology and treatment. Br J Biomed Sci 2001; 58: 101-10.

11.- Zangwill K, Hamilton D, Perkins B, Regnery R, Plikaytis B, Hadler J, et al. Cat scratch disease in Connecticut-epidemiology, risk factors, and evaluation of a new diagnostic test. N Engl J Med 1993; 329: 8-13.

12.- Ferrés M, Abarca K, Prado P, Montecinos L, Navarrete M, Vial P. Prevalencia de anticuerpos contra Bartonella henselae en niños, en adolescentes y en una población de riesgo ocupacional en Chile. Rev Méd Chile 2006; 134: 863-7.

13.- Ormerod L D, Dailey J P. Ocular manifestation of cat-scratch disease. Curr Opin Ophthalmol 1999; 10: 209-16.

14.- Wong M T, Dolan M J, Lattuada C P, Regnery R L, García M L, Mokulis E C, et al. Neuroretinitis, aseptic meningitis, and lymphadenitis associated with Bartonella (Rochalimaea) henselae infection in immunocompetent patients and patients infected with human immunodeficiency virus type 1 . Clin Infect Dis 1995; 21: 352-60.

15.- Ormerod L D, Skolnick K A, Menosky M M, Pavan P R, Pon D M. Retinal and choroidal manifestations of cat-scratch disease. Ophthalmology 1998; 105: 1024-31.

16.- Solley W A, Martin D F, Newman N J, King R, Callanan D G, Zacchei T, et. al. Cat scratch disease: posterior segment manifestations. Ophthalmology 1999; 106: 1546-53. 
17.- Täger M, Jahnsen J, Mediavilla M, Burgos R. Bartonelosis ocular: Reporte de tres casos. Rev Chil Infect 2008; 25: 58-63.

18.- Kerkhoff FT, Ossewaarde J M, de Loos W S, Rothova A. Presumed ocular bartonellosis. Br J Ophthalmol. 1999; 83: 270-5.

19.- Dreyer R F, Hopen G, Gass J D, Smith J L. Leber's idiopathic stellate neuroretinitis. Arch Ophthalmol 1984; 102: 1140-5.

20.- Esteve C, Achibet A M, Junot F, Ghassemi A, Rousseau B. A case of Leber's neuroretinitis. J Fr Ophtalmol 2000; 23: 794-6.

21.- Brazis P, Lee A. Optic disk edema with a macular star. Mayo Clin Proc 1996; 71: 1162-6.

22.- Ray S, Gragoudas E. Neuroretinitis. Int Ophthalmol Clin. 2001; 41: 83-102.

23.- Rappaport D, Cumming W, Ros P. Disseminated hepatic and splenic lesions in cat-scratch disease: imaging features. AJR. 1991; 156: 1227-8

24.- Garcia C J, Varela C, Abarca K, Ferres M, Prado P, Vial P. Regional lymphadenopathy in cat-scratch disease: ultrasonographic findings. Pediatr Radiol 2000; 30: 640-3.

25.- Florin T A, Zaoutis T E, Zaoutis L B. Beyond cat scratch disease: widening spectrum of Bartonella henselae infection. Pediatrics 2008; 121: e1413-25.
26.- Brenner S A, Rooney J A, Manzewitsch P, Regnery R L. Isolation of Bartonella (Rochalimaea) henselae: effects of methods of blood collection and handling. J Clin Microbiol. 1997 Mar; 35: 544-7

27.- Koehler J E, Sánchez M A, Garrido C S, Whitfeld M J, Chen F M, Berger T G, et al. Molecular epidemiology of Bartonella infections in patients with bacillary angiomatosis-peliosis. N Engl J Med 1997; 337: 1876-83.

28.- Martínez-Osorio H, Calonge M, Torres J, González F. Cat-scratch disease (ocular bartonellosis) presenting as bilateral recurrent iridocyclitis. Clin Infect Dis 2005; 40: e43-5

29.- Metzkor-Cotter E, Kletter Y, Avidor B, Varon M, Golan Y, Ephros M, et al. Long-term serological analysis and clinical follow-up of patients with cat-scratch disease. Clin Infect Dis 2003; 37 : 1149-54.

30.- Hansmann Y, DeMartino S, Piémont Y, Meyer N, Mariet P, Heller R, et. al. Diagnosis of cat scratch disease with detection of Bartonella henselae by PCR: a study of patients with lymph node enlargement. J Clin Microbiol 2005; 43: 3800-6.

31.- Rolain J M, Gourmet F, Enea M, Aboud M, Raoult D. Detection by immunofluorescence assay of Bartonella henselae in lymph nodes from patients with cat scratch disease. Clin Diagn Lab Immunol 2003; 10: 686-91.

32.- Fournier P E, Lelievre H, Eykyn S J, Mainardi J L, Marrie T J, Bruneel F, et al. Epidemiologic and clinical characteristics of Bartonella quintana and Bartonella henselae endocarditis: a study of 48 patients. Medicine (Baltimore) 2001; 80: 245-51.

33.- Rolain J M, Brouqui P, Koehler J E, Maguina C, Dolan M J, Raoult D. Recommendations for treatment of human infections caused by Bartonella species. Antimicrob Agents Chemother 2004; 48: 1921-33

34.- Margileth A M. Antibiotic therapy for catscratch disease: clinical study of therapeutic outcome in 268 patients and a review of the literature. Pediatr Infect Dis J 1992; 11: 474-8.

35.- Bass J W, Freitas B C, Freitas A D, Sisler C L, Chan D S, Vincent J M, et al. Prospective randomized double blind placebo-controlled evaluation of azithromycin for treatment of cat-scratch disease. Pediatr Infect Dis J 1998; 17: 447-52.

36.- Reed J B, Scales D K, Wong M T, Lattuada C P Jr, Dolan M J, Schwab I R. Bartonella henselae neuroretinitis in cat scratch disease. Diagnosis, management, and sequelae. Ophthalmology 1998; 105: 459-66. 